\title{
Circular dichroism in photoelectron angular distributions from two-color $(1+1)$ REMPI of NO
}

\author{
J. R. Applinga) and M. G. White \\ Department of Chemistry, Brookhaven National Laboratory, Upton, New York 11973 \\ R. L. Dubs \\ Arthur Amos Noyes Laboratory of Chemical Physics, ${ }^{\text {b) }}$ California Institute of Technology, Pasadena, \\ California 91125 \\ S. N. Dixit \\ Lawrence Livermore National Laboratory, L401, P.O. Box 808, Livermore, California 94550 \\ V. McKoy

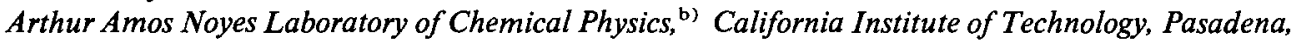 \\ California 91125
}

(Received 13 August 1987; accepted 10 September 1987)

\begin{abstract}
A detailed experimental and theoretical study of dichroic effects in photoelectron angular distributions is reported for $(1+1)$, two-color REMPI of NO via the $A^{2} \Sigma^{+}, v=0$ state. Optically aligned $A$ state rotational levels are probed through ionization by circularly polarized light. Resultant photoelectron angular distributions exhibit significant left-right asymmetry, the phase and magnitude of which are shown to be related to the curvature of the excited state $M_{J}$ distribution. Theoretical calculations involving a full $a b$ initio treatment of the ionization dynamics result in circularly dichroic angular distribution (CDAD) parameters in good agreement with those derived experimentally. Additional effects including hyperfine depolarization and coherence are also discussed in relation to the observed CDAD data.
\end{abstract}

\section{INTRODUCTION}

An $(n+m)$ resonantly enhanced multiphoton ionization (REMPI) process can be perceived as an $m$-photon ionization out of an aligned/oriented excited state created by the $n$-photon optical excitation. Atomic and molecular states can also be aligned by a variety of other methods including external fields, ${ }^{1,2}$ particle excitation, ${ }^{3}$ surface scattering, ${ }^{4}$ photodissociation, ${ }^{5}$ etc. In conventional methods for probing the state alignment, the anisotropy of either the fluorescence emitted by the state itself or the laser induced fluorescence (LIF) out the state is observed. ${ }^{6}$ More recently, a $(1+1)$ REMPI technique based on angle integrated cross sections has also been developed to probe ground state alignment. $^{\text {? }}$

Recently, the photoelectron angular distributions resulting from ionization out of an aligned state have been shown to exhibit a dichroic behavior; i.e., electron angutar distributions from aligned target states are different for photoionization with left or right circularly polarized light. This circular dichroism in angular distributions (CDAD) exists for nonchiral molecules, persists at the electric dipole approximation level, and is a direct signature of the state alignment. Predictions were made for the magnitude of the CDAD signal for adsorbed atoms and molecules, ${ }^{8,9}$ and also for gas-phase atoms and molecules whose state alignment is created by the absorption of linearly polarized photons. ${ }^{10}$ The first experimental demonstration of CDAD was recently reported. ${ }^{13}$

These theoretical and experimental CDAD studies have

\footnotetext{
a) Present address: Department of Chemistry, University of Kentucky, Lexington, KY 40506-0055

b) Contribution No. 7642 .
}

illustrated the magnitude of the effect and raise the possibility of employing CDAD as a technique for extracting the alignment of the initial state of the system. ${ }^{12}$ There are two features that strengthen this possibility. First is the factorization of the photoionization matrix elements out of the CDAD expressions and second is the ease of backtransforming the alignment of the resonant state to that of the initial state in an ( $n$ linear +1 circular)-type CDAD experiment.

As a prelude to this application, we have carried out an experimental and theoretical CDAD study of excitation-induced alignment of NO in $(1+1)$ REMPI via the $A^{2} \Sigma^{+}$, $v=0$ state. The two-color photoionization process utilized in this work can be expressed as a pump-probe sequence as follows:

$$
\begin{aligned}
\mathrm{NO}\left(X^{2} \Pi, v^{\prime \prime}\right. & \left.=0, J^{\prime \prime}\right)+\gamma(\sim 226 \mathrm{~nm} \text {, linearly polarized }) \\
& \rightarrow \mathrm{NO}^{*}\left(A^{2} \Sigma^{+}, v^{\prime}=0, J^{\prime}\right)
\end{aligned}
$$

$\mathrm{NO}^{*}\left(A^{2} \Sigma^{+}, v^{\prime}=0, J^{\prime}\right)+\gamma^{\prime}(266 \mathrm{~nm}$, circularly polarized $)$

$$
\rightarrow \mathrm{NO}^{+}\left(X^{1} \Sigma^{+}, v^{+}=0\right)+e^{-} \text {. }
$$

The pump radiation in process ( 1 ) is tuned to induce known rotational transitions and produce aligned populations of $A$ state $\left|J^{\prime} M_{J}\right\rangle$ substates $\left(M_{J}\right.$, is the space-fixed projection of total angular momentum $J^{\prime}$ ). This alignment is detected through subsequent ionization of $A$ state molecules with fixed-frequency, circularly polarized probe laser radiation. Photoelectron angular distributions are a measure of the photoelectron intensity variation as a function of the angle between the linear polarization vector of the pump beam and the electron detection axis. Circular dichroism in photoelectron angular distributions (CDAD) is determined as the difference between photoionizations carried out using left- 
and right-handed circularly polarized probe radiation.

\section{EXPERIMENTAL}

As shown schematically in Fig. 1, circular dichroism measurements require a pump-probe arrangement of two laser beams with independently controllable polarizations. The tunable laser radiation used for the excitation (pump) step was obtained by nonlinear mixing (Quanta Ray WEX) of the frequency doubled output from a dye laser (Quanta Ray PDL) with residual IR photons from the pulsed (20 $\mathrm{Hz}$ ) Nd:YAG pump laser (Quanta Ray DCR). Output powers of up-mixed dye laser radiation at $226 \mathrm{~nm}$ are typically in the range $0.5-1.5 \mathrm{~mJ} /$ pulse using a mixture of $\mathrm{R} 590$ and R610 laser dyes (Exciton) in a ratio of approximately 10:1. The ionization (probe) laser beam is generated simultaneously by the same laser system. A $5 \%$ beam splitter inserted into the dye laser directs a portion of the $532 \mathrm{~nm}$ Nd:YAG output through telescopic optics to a frequency doubling crystal cell (in rad) which produces a beam at 266 $\mathrm{nm}$ with powers in the range $1-2 \mathrm{~mJ} /$ pulse.

Both laser beam outputs are linearly polarized greater than $95 \%$; however, each beam passes through a Glan polarizing prism to ensure $100 \%$ polarization purity before the polarization vectors are modified further. The polarization vector of the tunable dye laser radiation is rotated with a Soleil-Babinet (Karl Lambrecht) compensator tuned to $1 / 2$ wave retardation at the wavelength of interest. The probe laser beam is passed through a $1 / 4$ wave plate (CVI) which converts linearly polarized $266 \mathrm{~nm}$ radiation to circularly polarized radiation when the axis of the $1 / 4$ wave plate is at an angle of $45^{\circ}$ with respect to the incoming polarization vector. The axis of the $1 / 4$ wave plate can occupy two inequivalent positions corresponding to production of oppositehanded circular polarization designated "left" and "right." The pump beam is admitted to the photoelectron spectrometer chamber unfocused and the counterpropagating probe beam is focused through a $250 \mathrm{~nm}$ focal length lens mounted on a translation stage to allow adjustable beam overlap for maximum two-color $(1+1)$ REMPI signal.

A complete description and illustration of the photoelectron spectrometer system can be found in Ref. 14. In the present investigation, two different sample introduction methods were utilized to admit nitric oxide target molecules to the interaction region of the spectrometer system. To reduce the congestion of the $A$ state $(v=0)$ rotational spectrum, angular distribution measurements were performed on rotationally "cold" NO produced by high pressure expansions ( $40 \mathrm{psi}$ ) of a $4 \%$ mixture of NO (Matheson, $99.6 \%$ purity) in argon. The chamber pressure was typically 0.6 $1.5 \times 10^{-5}$ Torr for pulsed molecular beam valve (Newport Corp. BV 1000) operation in the PES apparatus. The NO/ Ar gas pulses enter the interaction region at right angles to both the propagation direction of the laser and the detector axis of the spectrometer flight tube. Measurements for transitions of high $J^{\prime \prime}$ or of ${ }^{2} \Pi_{3 / 2}$ ground state ( $P_{12}$ branch) molecules were performed with neat, room temperature NO gas samples admitted through an effusive nozzle ( $70 \mu \mathrm{m}$ diameter) attached below the interaction region and facing the $1.5 \mathrm{~mm}$ aperture entrance to the PES flight tube.

Circular dichroism measurements of photoelectron angular distributions are the result of the difference between an experiment performed with the probe laser converted to lefthanded polarization and a subsequent scan taken with righthanded circular polarization. Photoelectrons ejected at right angles to the counterpropagating pump/probe laser beams were collected $\left(\sim 1 \times 10^{-3} \mathrm{sr}\right)$ as a function of the angle $(\theta)$ between the linear polarization vector of the pump beam and the detector axis of the TOF spectrometer. A photodiode triggered by the dye laser pulse provides a time zero for the photoelectron time of flight. The TOF photoelectron spectrum presented in Fig. 2 shows the relative amounts of twocolor $(1+1)$ and one-color $(1+1)$ ionization when the dye laser polarization is parallel to the detector axis. The
2) PROBE OF ALIGNMENT

$$
\lambda^{\prime}=266 \mathrm{~nm}
$$

left or right

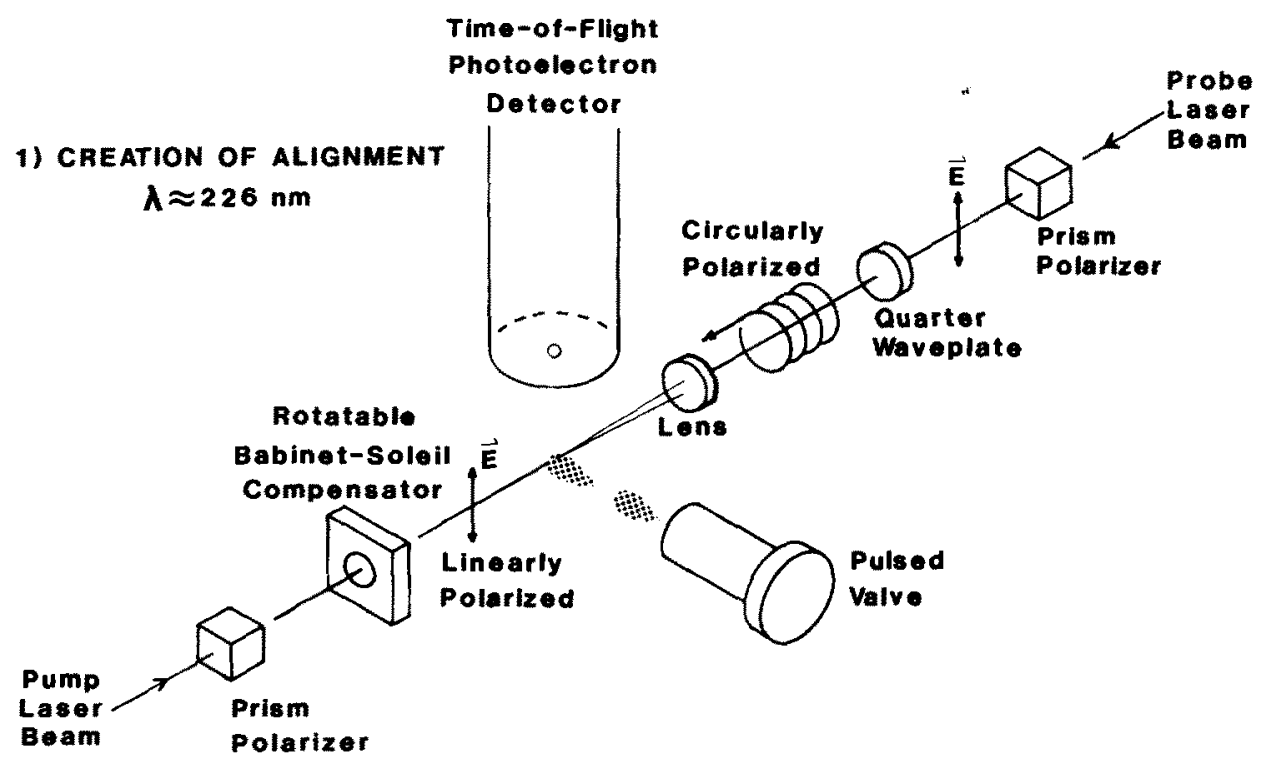

FIG. 1. Schematic of the experimental setup. Individual components described in the text. 


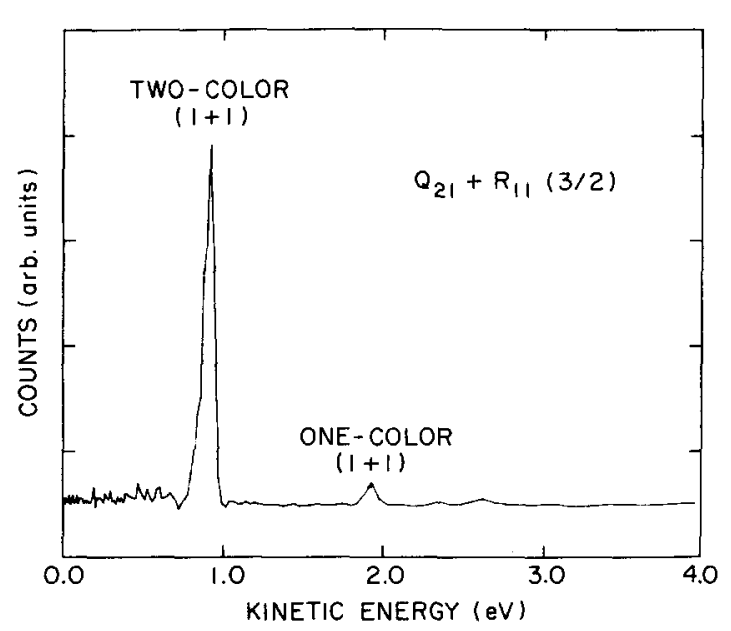

FIG. 2. Photoelectron spectrum resulting from $(1+1)$, one- and two-color REMPI of NO via the $Q_{21}+R_{11}(3 / 2)$ rotational branch of the $A, v^{\prime}=0-X, v^{\prime \prime}=0$ transition. Both laser beams were linearly polarized with their polarization directions at $\theta=0^{\circ}$. The difference in kinetic energies between the photoelectron peaks correspond to the energy difference between the 266 (two-color) and $226.3 \mathrm{~nm}$ (one-color) ionizing photons.

Rydberg character of the $A$ state is manifested by the existence of a single photoelectron peak corresponding to production of $v^{+}=0$ states of the $\mathrm{NO}^{+}$ion. Thus, the angular dependence is determined by monitoring the REMPI photoelectrons in the two-color $v^{+}=0$ channel for a specific rotational transition as a function of pump laser polarization angle determined by the Soleil-Babinet compensator. Data was accumulated for 50 laser shots for each angle incremented by $10^{\circ}$ during 12 scans of $360^{\circ}$. Collection of the digitized output of the multichannel plate photoelectron detector and control of Soleil-Babinet stage rotations were accomplished through the use of CAMAC molecules (Kinetic Systems 3912 Crate Controller, LeCroy 8828 Transient Recorder, Kinetic Systems 3112 12-bit DAC) interfaced to a PDP 11/ 73 minicomputer.

Angular scans were taken under similar conditions for both left- and right-handed probe polarizations. The first and last $180^{\circ}$ segments of the $360^{\circ}$ scans were averaged, three-point smoothed and normalized to the cross section at $90^{\circ}$ before the difference was taken between left- and righthanded data. The resultant CDAD curves were fit analytically to associated Legendre polynomials to extract coefficients indicative of the excited state alignment.

\section{THEORY}

\section{A. Alignment}

In the present experiment, the ground state molecules are distributed isotropically, i.e., all the $M_{J}$ sublevels for a given $J^{\prime \prime}$ level have the same population. However, after one photon absorption of linearly polarized light, the substates of different values of $\left|M_{J}\right|$ acquire different populations, resulting in an "aligned" excited state. ${ }^{6}$ The details of this alignment depend on the excitation branch $(P, Q$, or $R)$ and are reflected qualitatively by the sign of the state multipole moment $A_{2}$ (Fig. 3 ). Note that a $J^{\prime}=1 / 2$ state cannot support any alignment $\left(A_{2}=0\right)$ since the $M_{J}=1 / 2$ and

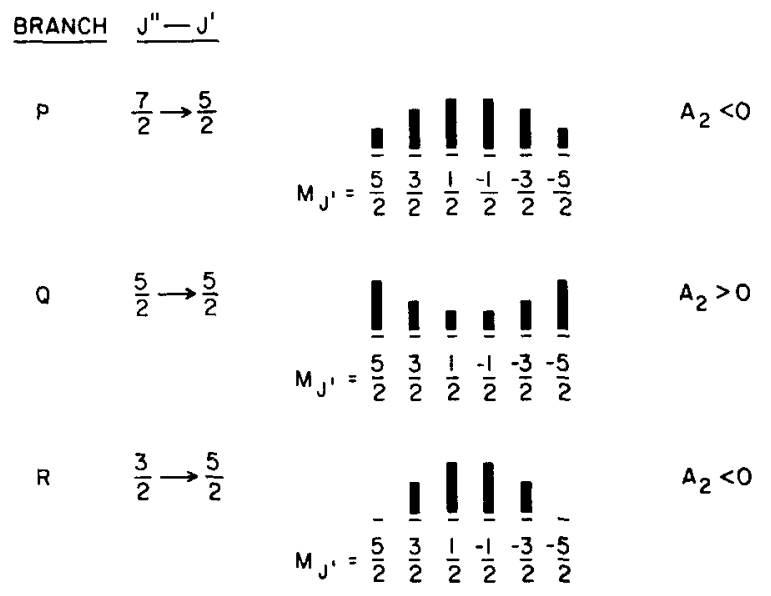

FIG. 3. Relative $M_{J}$ population distributions for an upper $J^{\prime}=5 / 2$ level populated via one-photon $P, Q$, and $R$ transitions. $A_{2}$ is the quadrupole moment of the alignment as described in the text.

$M_{J}=-1 / 2$ sublevels will always have the same population. For one photon excitation from an isotropic initial state with linearly polarized light, the relative populations $N\left(M_{J^{\prime}}\right)$ of the excited state are given by ${ }^{15}$

$$
N\left(M_{J^{\prime}}\right) \propto\left(\begin{array}{lll}
J^{\prime} & 1 & J^{\prime \prime} \\
M_{J^{\prime}} & 0 & -M_{J^{\prime \prime}}
\end{array}\right)^{2},
$$

where $J$ " represents the total angular momentum of the initial state and $J^{\prime}$ that of the excited state. The conversion from the $N\left(M_{J}\right)$ description of alignment to that of $A_{L}$ is straightforward. ${ }^{6}$

\section{B. CDAD}

The CDAD intensity, defined as $I_{\mathrm{CDAD}}(\theta)=$ $I_{\mathrm{LEFT}}(\theta)-I_{\mathrm{RIGHT}}(\theta)$, is given by ${ }^{11}$

$$
I_{\mathrm{CDAD}}(\theta)=\sum a_{L} P_{L}^{1}(\cos \theta),
$$

where

$$
a_{L}=A_{L} \overline{\bar{\beta}}_{L} .
$$

$A_{L}$ are the state multipole moments of the alignment for the resonant state, $P_{L}^{1}(\cos \theta)$ are associated Legendre polynomials, and $\theta$ is the angle between the pump photon polarization vector and the electron collection direction. All information concerning the photoionization dynamics are incorporated in $\overline{\bar{\beta}}_{L}$. The fact that the moment index $L$ of the alignment $\left(A_{L}\right)$ is the same as that for the photoelectron $\left(\overline{\bar{\beta}}_{L}\right)$ is unique to CDAD and is not true for typical photoelectron angular distributions. It is this fact that makes CDAD so useful for studying alignment.

In general, the alignment of a state is characterized by even moments $A_{0}, A_{2}, A_{4}$, etc. However, for one photon excitation from an unaligned ground state, only $A_{0}$ and $A_{2}$ are nonzero. Since $A_{0}$ does not contribute to the alignment ( $A_{0}$ relates only to the total population of the state ${ }^{6}$ ) the CDAD intensity reduces to

$$
\begin{aligned}
I_{\mathrm{CDAD}}(\theta) & =A_{2} \overline{\bar{\beta}}_{2} P_{2}^{1}(\cos \theta) \\
& =\frac{3}{2} A_{2} \overline{\bar{\beta}}_{2} \sin 2 \theta .
\end{aligned}
$$

The $J$ dependence of $\bar{\beta}_{L}$ can be factored out as 


$$
\bar{\beta}_{L}\left(J^{\prime}\right)=X_{L}\left(J^{\prime}\right) \cdot \tilde{\beta}_{L},
$$

where $\tilde{\beta}_{L}$ is explicitly independent of $J^{\prime}$; it depends on $J^{\prime}$ only through the variation of the cross section with the photoelectron kinetic energy. Because rotational spacings are small, we can ignore this latter dependence and treat $\tilde{\beta}_{L}$ as a constant. $X_{2}\left(J^{\prime}\right)$ has a simple expression in $J^{\prime}$, the value of which quickly approaches a high $J^{\prime}$ limit of $-\sqrt{\left(J^{\prime} / 2\right)} .^{16}$ The important point here is that all $\bar{\beta}_{L}$ (for any value of $J^{\prime}$ ) have the same sign, so that any change in the sign of $A_{2} \bar{\beta}_{2}$ is due to a change in the sign of the moment $A_{2}$ (ignoring coherence effects).

\section{Normalization of the CDAD spectra}

The experimental and theoretical values of $A_{2} \overline{\bar{\beta}}_{2}$ presented in this work are all normalized by dividing the raw value by the intensity of the left or right spectrum at $90^{\circ}$ $\left[I_{L, R}\left(90^{\circ}\right)\right]$. In this way the relative magnitude of the CDAD signal to the left or right photoionization signal can be obtained. However, the photoionization dynamics and alignment are intimately entangled in the value of $I_{L, R}\left(90^{\circ}\right)$. (The alignment dependence can be seen in Fig. 2 of Ref. 10.) Nevertheless, a semiquantitative statement can be made about the trends in the $A_{2} \overline{\bar{\beta}}_{2}$ values reported in this fashion. The value of $I_{L, R}\left(90^{\circ}\right)$ can be written

$$
I_{L, R}\left(90^{\circ}\right)=c_{0} A_{0}+c_{2} A_{2},
$$

where $c_{0}$ and $c_{2}$ depend on the photoionization dynamics and on $J^{\prime}$. Generally, the leading term $c_{0} A_{0}$ dominates the expression and so we can write

$$
I_{L, R}\left(90^{\circ}\right) \sim c_{0} A_{0}
$$

from which it follows that

$$
\frac{A_{2} \overline{\bar{\beta}}_{2}}{I_{L, R}\left(90^{\circ}\right)} \propto\left[\frac{\left(2 J^{\prime}-1\right)\left(2 J^{\prime}+3\right)}{J^{\prime}\left(J^{\prime}+1\right)}\right]^{1 / 2} \cdot \frac{A_{2}}{A_{0}},
$$

where the explicit $J^{\prime}$ dependent part of the above expression is that for the ratio $\left(\bar{\beta}_{2} / c_{0}\right) .{ }^{16}$ Indeed, if one plots the theoretical values of the left-hand side of Eq. (9) vs those of the right-hand side for all the clean branches given in this paper, a graph very close to a straight line is obtained (Fig. 4). Note that this approximation cannot be applied to the mixed branches. For one photon excitation with linearly polarized light from an unaligned initial state the following relations hold:

$P$ branch:

$$
\frac{A_{2}}{A_{0}}=-\left[\frac{\left(2 J^{\prime \prime}-3\right)\left(J^{\prime \prime}-1\right)}{5\left(2 J^{\prime \prime}+1\right) J^{\prime \prime}}\right]^{1 / 2}
$$

$R$ branch:

$$
\frac{A_{2}}{A_{0}}=-\left[\frac{\left(2 J^{\prime \prime}+5\right)\left(J^{\prime \prime}+2\right)}{5\left(2 J^{\prime \prime}+1\right)\left(J^{\prime \prime}+1\right)}\right]^{1 / 2} .
$$

The right-hand side of Eq. (9) is directly proportional to the alignment parameter $A_{0}^{(2)}$ commonly used in LIF studies. ${ }^{5.6}$

\section{Coherence}

Mixed branches such as $Q_{21}+R_{11}$ offer two indistinguishable pathways (within the resolution of this experiment) to ionization. In addition to direct contributions to

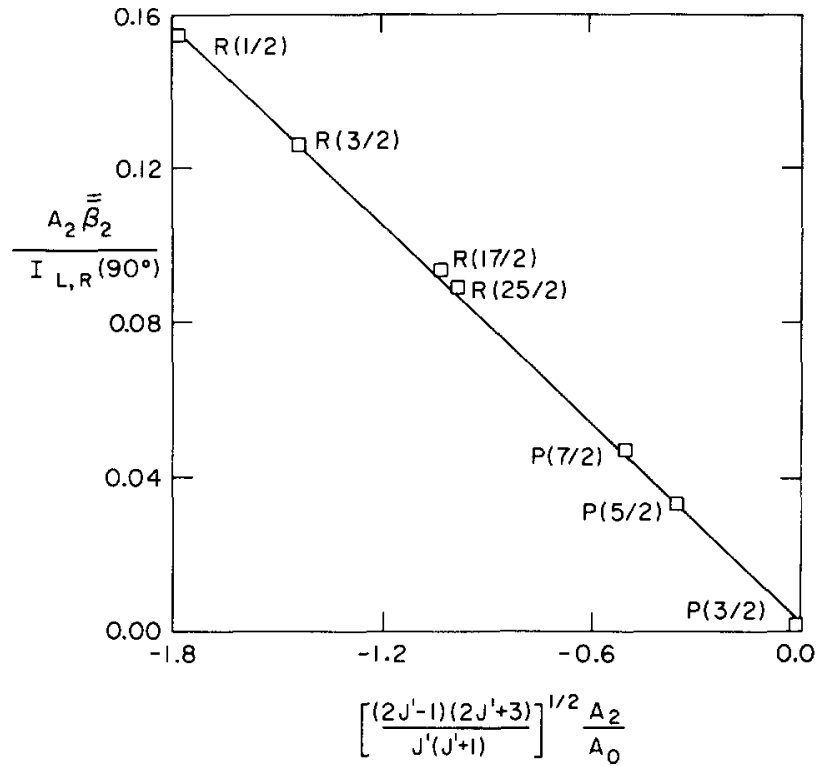

FIG. 4. Graphical representation of the direct relationship between the CDAD intensity normalized at $\theta=90^{\circ}$ and the relative moments of the excited state alignment for one photon excitation with linearly polarized light [see Eq. (9) in the text].

the photoelectron signal by each branch individually, a coherent contribution arising from interference between the pathways can also contribute to the signal. The transition probability then involves a coherent sum over these two paths

Transition probability

$$
\begin{aligned}
& =\left|R_{\text {path }}+Q_{\text {path }}\right|^{2} \\
& =\left|R_{\text {path }}\right|^{2}+\left|Q_{\text {path }}\right|^{2}+2 \operatorname{Re}\left(R_{\text {path }} Q_{\text {path }}^{*}\right),
\end{aligned}
$$

where the last term in Eq. (12b) represents the interference of the $R$ and $Q$ excitation paths. Whether or not this coherence contributes depends on the energy splitting $\Delta E$ between the unresolved intermediate states. The coherence lifetime $\left(\tau_{\text {coh }}=\hbar / \Delta E\right)$ associated with this splitting must be longer than the apparent "lifetime" of the state (in this case the excited state is ionized within the laser pulse duration $\sim 10$ ns). If $\tau_{\text {coh }}$ is shorter than this time, the coherence dies away before the intermediate state is probed.

\section{E. Calculations}

The details for obtaining the value of $A_{2} \bar{\beta}_{2}$ are given in Refs. 10 and 11. The calculations "with hyperfine" include long-time limit, average hyperfine depolarization as described in Ref. 6. For mixed branches, the spectra for the individual branches are weighted by the relative line strengths given in Ref. 17. The coherence effects are treated in the two extreme limits; one in which complete coherence persists and the other wherein the coherence is time averaged out.

\section{RESULTS AND DISCUSSION}

The CDAD results obtained in this investigation of twocolor $(1+1)$ photoionization of NO are shown in Figs. 5-7 


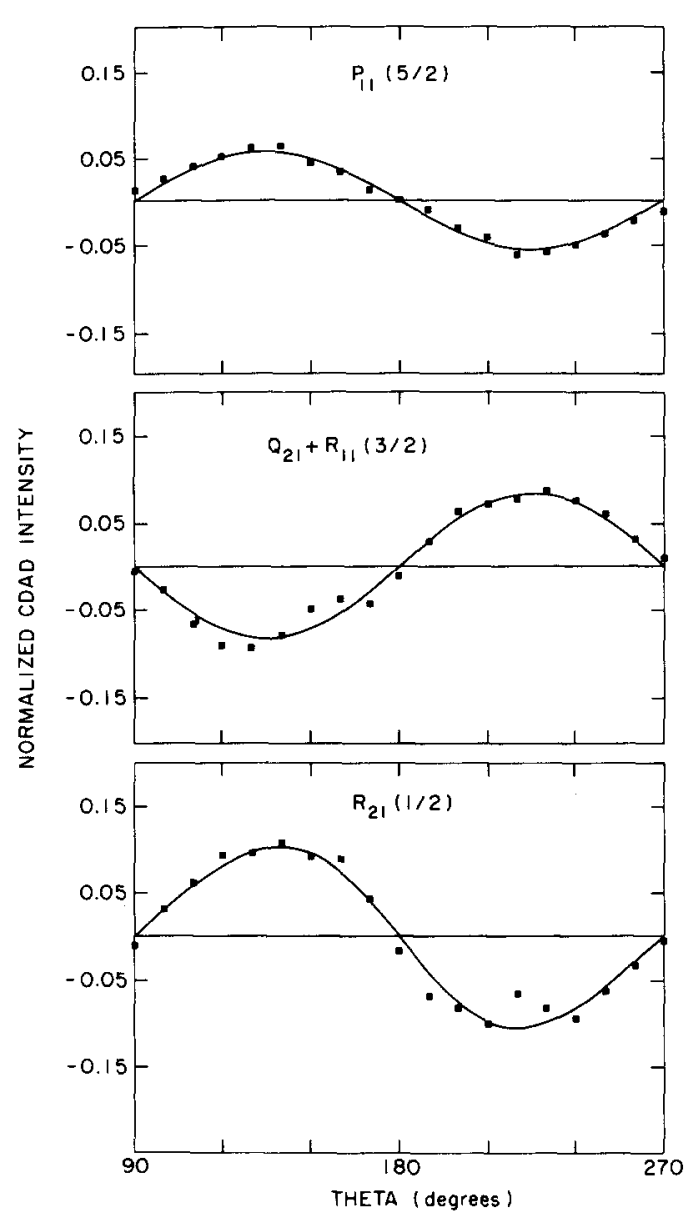

FIG. 5. Experimental CDAD for three rotational transitions which lead to $J^{\prime}=3 / 2\left[Q_{21}(3 / 2)\right.$ contribution dominates the $R_{11}(3 / 2), J^{\prime}=5 / 2$ component ]. Experimental data; - - - least-squares fit to Eq. (5).

and summarized in Table I. The alignment parameters $A_{2} \overline{\bar{\beta}}_{2}$ listed Table I for each transition are extracted as coefficients of an analytical least-squares fit of the angular distribution data to the associated Legendre polynomials series of Eq. (4a). The coefficients are normalized to the intensity of the left or right spectrum at $90^{\circ}$. Coefficient errors listed are statistical errors determined by the analytical fit and do not

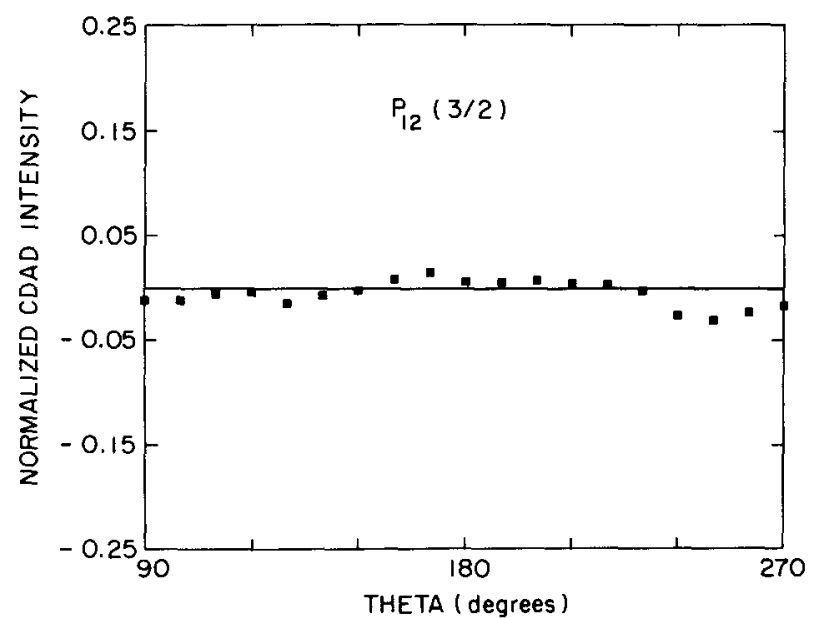

FIG. 6. Experimental CDAD for the $P_{11}(3 / 2)$ rotational transition leading to the isotropic $J^{\prime}=1 / 2$ level. Experimental data; - - - least-squares fit to Eq. (5).

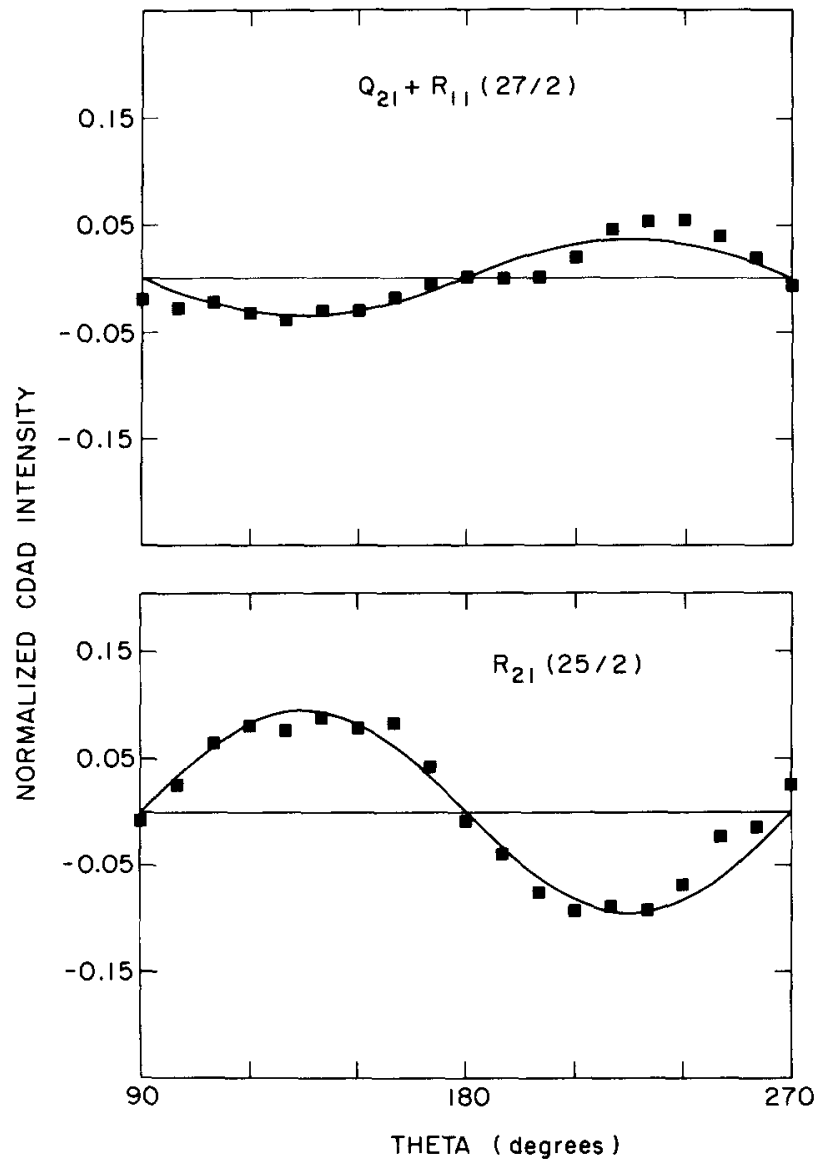

FIG. 7. Experimental CDAD for two rotational transitions leading to high $J^{\prime}$. $\square$ Experimental data; - . - least-squares fit to Eq. (5).

represent confidence level or reproducibility.

Theoretical values for $A_{2} \bar{\beta}_{2}$ are also listed in Table I. The "theory with hyperfine" values have been calculated for low $J$ only since hyperfine effects are negligible at higher $J .^{6}$ These latter values represent the average depolarization one would expect in the long-time limit. However, in these experiments the exicted molecules are ionized after 5-10 ns. Because the hyperfine precession occurs on the same time scale, ${ }^{18}$ a rigorous investigation of the extent of depolarization would require a more sophisticated dynamical treatment. The theory with hyperfine values then should be interpreted as long time limit values which would be completely attained if the time delay between pump and probe laser pulses was increased.

The phases of the experimental and theoretical $A_{2} \bar{\beta}_{2}$ are clearly branch dependent as seen in Table I. Because $A_{2} \bar{\beta}_{2}$ values for the mixed branches are opposite in sign from the clean $P$ and $R$ branches, the experimental data indicate that the mixed branches are dominated by the $Q$ component, a fact which is supported by calculation [the anomalous $Q_{21}+R_{11}(1 / 2)$ result will be addressed later]. The opposite phase for the $Q$ branch is most clearly seen in Fig. 5, in which all three spectra arise from the same $J^{\prime}=3 / 2$ intermediate state. This opposite phase for the $Q$ branch is a direct result of the opposite alignment shown in Fig. 3.

In terms of the magnitudes of the $A_{2} \overline{\bar{\beta}}_{2}$ values, the experimental data fall into two groups relative to the theoretical values. The first group is comprised of the low $J^{\prime \prime}$ values, 
TABLE I. Summary of results for experimental and theoretical CDAD parameters for $(1+1)$, two-color REMPI of NO through various rotational transitions.

\begin{tabular}{lrccc}
\hline \hline Branch & \multicolumn{1}{c}{$\begin{array}{c}\text { Experimenta } \\
\boldsymbol{A}_{2} \bar{\beta}_{2}\end{array}$} & $\begin{array}{c}\text { Theory } \\
A_{2} \bar{\beta}_{2}\end{array}$ & $\begin{array}{c}\text { Theory with hyperfine } \\
A_{2} \bar{\beta}_{2}\end{array}$ \\
\hline$P_{11}$ & $3 / 2$ & $+0.004(0.005)$ & 0 & 0 \\
$P_{11}$ & $5 / 2$ & $+0.038(0.005)$ & +0.033 & +0.010 \\
$P_{11}$ & $7 / 2$ & $+0.036(0.006)$ & +0.047 & +0.029 \\
$P_{12}$ & $3 / 2$ & $-0.001(0.003)$ & 0 & 0 \\
$P_{12}$ & $5 / 2$ & $+0.035(0.002)$ & +0.033 & +0.010 \\
$P_{12}$ & $7 / 2$ & $+0.028(0.004)$ & +0.047 & +0.029 \\
$Q_{11}+P_{21}$ & $31 / 2$ & $-0.060(0.004)$ & -0.136 & \\
$Q_{11}+P_{21}$ & $33 / 2$ & $-0.069(0.003)$ & -0.137 & \\
$Q_{21}+R_{11}$ & $1 / 2$ & $-0.050(0.008)$ & $+0.054^{\mathrm{c}}$ & +0.016 \\
$Q_{21}+R_{11}$ & $3 / 2$ & $-0.056(0.008)$ & $-0.034^{\mathrm{d}}$ & +0.006 \\
$Q_{21}+R_{11}$ & $27 / 2$ & $-0.024(0.002)$ & -0.032 & \\
$R_{21}$ & $1 / 2$ & $+0.070(0.003)$ & +0.155 & +0.048 \\
$R_{21}$ & $3 / 2$ & $+0.069(0.004)$ & +0.126 & +0.078 \\
$R_{21}$ & $17 / 2$ & $+0.058(0.003)$ & +0.093 & \\
$R_{21}$ & $25 / 2$ & $+0.063(0.003)$ & +0.089 & \\
\hline
\end{tabular}

${ }^{a}$ Normalized to the differential cross section at $\theta=90^{\circ}$. Tabulated coefficients represent averages determined from all experiments performed on each line. Errors given in parentheses only represent the statistical accuracy of the parameters extracted from the fitting procedure.

'Only calculated for low $J$ ".

c -0.182 with coherence.

d -0.139 with coherence.

which lie in general between the theoretical values with and without hyperfine depolarization. For the $P_{11}(3 / 2)$ and $P_{12}(3 / 2)$ branches, which both access a $J^{\prime}=1 / 2$ intermediate state, $A_{2} \bar{\beta}_{2}$ is found to be zero within experimental error since this intermediate state cannot support any alignment. The experimental CDAD spectrum for the $P_{12}(3 / 2)$ branch is shown in Fig. 6. It is apparent from Table I and Fig. 5 that the $A_{2} \bar{\beta}_{2}$ values for the $P$ branches are smaller than those for $R$ branches. It should also be noted that in the weak field regime, the clean $P_{11}\left(J^{\prime \prime}\right)$ and $P_{12}\left(J^{\prime \prime}\right)$ branches yield identical CDAD which reflects the independence of the $A_{2} / A_{0}$ on the fine structure component of the ground state. These results are predicted by Eqs. (10) and (11).

The second group of data is comprised of high $J$ " results in which the experimental $A_{2} \overline{\bar{\beta}}_{2}$ values are systematically lower than the theoretical values. The origin of this quantitative discrepancy is not clear at this time. Nevertheless, both the experimental and theoretical results indicate that the $Q_{21}+R_{11}(27 / 2) A_{2} \overline{\bar{\beta}}_{2}$ value is much lower than the other values at high $J^{\prime \prime}$. The reason for this result is straightforward. The $R_{21}(17 / 2)$ and $R_{21}(25 / 2)$ branches are "clean" excitations while the $Q_{11}+P_{21}(31 / 2)$ and $Q_{11}+P_{21}(33 / 2)$ excitations have a $Q$ component with a line strength roughly five times that of the $P$ component. ${ }^{17}$ The $Q$ and $R$ components of the $Q_{21}+R_{11}(27 / 2)$ transition, on the other hand, have roughly the same line strengths. ${ }^{17}$ Because $Q$ and $R$ branches have opposite CDAD phases, the CDAD spectrum for this mixed branch is almost completely annihilated, with a small amount of $R$ character surviving. CDAD spectra for the $Q_{21}+R_{11}(27 / 2)$ and $R_{21}(25 / 2)$ lines are shown in Fig. 7.

The most surprising $A_{2} \bar{\beta}_{2}$ value obtained is that for the
$Q_{21}+R_{11}(1 / 2)$ transition. The experimental phase is opposite that of theory. For this mixed line, the $Q$ component accesses $J^{\prime}=1 / 2$ in the intermediate state while the $R$ component accesses $J^{\prime}=3 / 2$. The $Q$ component cannot contribute directly to CDAD since it leads to a $J^{\prime}=1 / 2$ upper state which cannot support any alignment. For this reason, one would predict the $Q_{21}+R_{11}(1 / 2)$ line to have an $A_{2} \overline{\bar{\beta}}_{2}$ value with the same phase as the clean $R$ branches. However, experimentally the opposite phase is found. Various experimental conditions were changed in an attempt to eliminate possible artifacts which could give rise to this reverse phase effect, e.g., laser pulse duration ( 10 to $2 \mathrm{~ns}$ ), laser beam intensities, focal volumes, and external fields. Under all conditions the CDAD remained unchanged. A linear dependence of the integrated two-color $(1+1)$ photoelectron signal on pump laser power also verified that the measurements were not made in a saturated power regime.

We attribute the anomalous CDAD phase to coherent excitation of the intermediate state. The coherent lifetime $\tau_{\text {coh }}$ associated with the $J^{\prime}=1 / 2,3 / 2$ energy splitting is approximately $\sim 45 \mathrm{~ns}$. Clearly, coherence can survive the time delay between excitation and ionization. Therefore, while the $Q$ component of the $Q_{21}+R_{11}(1 / 2)$ branch cannot contribute directly to the CDAD spectrum, it can contribute by interfering with the $R$ component. The interference contribution in Eq. (12) is calculated to have the opposite phase and a larger magnitude than the direct $R$ contribution as indicated in Table I.

As $J^{\prime}$ increases the splitting between the energy levels increases and $\tau_{\text {coh }}$ decreases. For $N=1, J^{\prime}=3 / 2,5 / 2, \tau_{\text {coh }}$ is found to be $\sim 27 \mathrm{~ns}$. In this case, coherence can contribute to the $Q_{21}+R_{11}(3 / 2)$ transition, although the $Q$ contribu- 
tion is already dominant without coherence. At high $J^{\prime}$, however, the coherent lifetime becomes too short for coherence to affect the CDAD spectra; e.g., at $J^{\prime}=29 / 2,31 / 2, \tau_{\text {coh }} \approx 4$ ns. Note that with complete coherence included, the experimental $A_{2} \beta_{2}$ values for the $Q_{21}+R_{11}(1 / 2)$ and (3/2) transitions fall between the theoretical values with and without hyperfine depolarization. (The with hyperfine results are long time limit results in which the coherence is assumed to have died away.)

The presence of coherence seems to be the only plausible explanation for the $Q_{21}+R_{11}(1 / 2)$ result. Coherence might be studied further by examining the magnitude and phase of the CDAD spectrum for this mixed branch as a function of the delay time between pump and probe pulses. Such experiments will be the focus of future investigations.

\section{CONCLUSIONS}

The role of the CDAD method in determination of excited state alignment has been firmly established by this and the previous study for REMPI-PES of nitric oxide. ${ }^{13}$ CDAD has the same characteristic as fluorescence anisotropy measurements in LIF in that it provides a direct positive identification of excited state alignment just by the existence of a dichroic signal. CDAD studies are of more general applicability than LIF since the target levels are not required to be connected to fluorescing states for detection. The ability of photoelectron angular distributions to probe higher moments of the excited state alignment $(L \geqslant 4)$ is an additional feature of CDAD measurements. ${ }^{11,16}$ At present, however, the CDAD technique is more experimentally demanding than fluorescence methods due to the smaller inherent collection solid angle of the electron analyzer and the necessity for two laser beams with independently controlled polarizations.

The $A_{2} \overline{\bar{\beta}}_{2}$ values obtained from CDAD measurements contain information about excited state alignment in both their phase and magnitude; the former relates to the shape of the excited state $M_{J}$ distribution and the latter relates to the degree of the alignment. The possibility of probing coherent excitation with CDAD is suggested by some of results presented here.

The present studies provide a foundation for extensions of the CDAD technique to the study of ground state alignment induced by chemical processes. In particular, the usefulness of $(1+1) \mathrm{CDAD}$ as a probe of photofragment alignment was addressed in a recent paper. ${ }^{12}$ That work showed that if the $A_{2} \overline{\mathcal{\beta}}_{2}$ values for two different branches arising from the same $J$ " were compared, the initial state alignment can be obtained independent of the photoionization dynamics. Experimental studies of ground state alignment induced by photofragmentation of molecular precursors containing weakly bound $\mathrm{NO}$ (e.g., $\mathrm{CH}_{3} \mathrm{ONO}$ ) are currently in progress.

\section{ACKNOWLEDGMENTS}

The research at the Brookhaven National Laboratory was supported under Contract No. DE-AC02-76CH00016 with the U. S. Department of Energy and by its Division of Chemical Sciences, Office of Basic Energy Sciences. Work at the California Institute of Technology was supported by grants from the National Science Foundation (CHE8521391), Air Force Office of Scientific Research (Contract No. 87-0039), and the Office of Health and Environmental Research of the U. S. Department of Energy (DE-FG0387ER60513). R.L.D. and V.M. also acknowledge the use of the resources of the San Diego Super Computer Center, which is supported by the National Science Foundation. Research at Lawrence Livermore National Laboratory was performed under Contract No. W-7405-ENG-48 with the U. S. Department of Energy.

'J. Reuss, Adv. Chem. Phys. 30, 389 (1975).

${ }^{2}$ P. R. Brooks, Science 193, 11 (1976).

${ }^{3}$ R. Bersohn and S. H. Lin, Adv. Chem. Phys. 16, 67 (1969).

${ }^{4}$ M. C. Lin and G. Ertl, Annu. Rev. Phys. Chem. 37, 587 (1986), and references therein.

${ }^{5}$ Recent representative papers include R. Vasudev, R. N. Zare, and R. N. Dixon, J. Chem. Phys. 80, 4863 (1984); G. E. Hall, N. Sivakumar, and P. L. Houston, ibid. 84, 2120 (1986); M. Dubs, U. Bruhlmann, and J. R. Huber, ibid. 84, 3106 (1986); F. Lahmani, C. Lardeux, and D. Solgadi, Chem. Phys. Lett. 129, 24 (1986).

${ }^{6}$ C. H. Greene and R. N. Zare, Annu. Rev. Phys. Chem. 33, 119 (1982); J. Chem. Phys. 78, 6741 (1983).

${ }^{7}$ D. C. Jacobs and R. N. Zare, J. Chem. Phys. 85, 5457 (1986); D. C. Jacobs, R. J. Madix, and R. N. Zare, ibid. 85, 5469 (1986).

${ }^{8}$ R. L. Dubs, S. N. Dixit, and V. McKoy, Phys. Rev. Lett. 54, 1249 (1985).

${ }^{9}$ R. L. Dubs, S. N. Dixit, and V. McKoy, Phys. Rev. B 32, 8389 (1985).

${ }^{10}$ R. L. Dubs, S. N. Dixit, and V. McKoy, J. Chem. Phys. 85, 656 (1986).

${ }^{11}$ R. L. Dubs, S. N. Dixit, and V. McKoy, J. Chem. Phys. 85, 6267 (1986).

${ }^{12}$ R. L. Dubs, S. N. Dixit, and V. McKoy, J. Chem. Phys. 86, 5886 (1987).

${ }^{13}$ J. R. Appling, M. G. White, T. M. Orlando, and S. L. Anderson, J. Chem. Phys. 85, 6803 (1986).

${ }^{14}$ J. R. Appling, M. G. White, W. J. Kessler, R. Fernandez, and E. D. Poliakoff, J. Chem. Phys. (to be published).

${ }^{15}$ S. N. Dixit, D. L. Lynch, V. McKoy, and W. M. Huo, Phys. Rev. A 32, 1267 (1985).

${ }^{16}$ R. L. Dubs, S. N. Dixit, and V. McKoy, J. Chem. Phys. (to be published).

${ }^{17}$ L. T. Earls, Phys. Rev. 48, 423 (1935).

${ }^{18}$ T. Bergeman and R. N. Zare, J. Chem. Phys. 61, 4500 (1974). 